\title{
Una nueva definición de la logística interna y forma de evaluar la misma
}

\author{
A new definition of internal logistics and how to evaluate it \\ Orlem Pinheiro de Lima ${ }^{1 *} \quad$ Sandro Breval Santiago ${ }^{2}$ \\ Carlos Manuel Rodríguez Taboada ${ }^{3} \quad$ Neimar Follmann ${ }^{3}$ \\ Recibido 22 de octubre de 2015, aceptado 26 de julio de 2016 \\ Received: October 22, $2015 \quad$ Accepted: July 26, 2016
}

\begin{abstract}
RESUMEN
El mercado ha requerido de las organizaciones, eficiencia, eficacia, creatividad y agilidad, para ser competitivo y definir sus estrategias encaminadas a la sostenibilidad del negocio. En este contexto, la logística se perfila como un eslabón importante en las estrategias corporativas. Sin embargo, en la lectura previa sobre este tema, hay una laguna acerca de la organización y evaluación de la logística interna. Buscando llenar este vacío, este trabajo tiene como objetivo identificar la importancia de evaluar la logística interna, identificando sus partes componentes, cómo se estructuran y se miden, basados en la literatura y en respuestas de expertos en este campo. El grado de importancia de estas partes será evaluado por tres empresas, mediante una escala Likert. Se muestra la identificación de las partes componentes de la logística interna, los pesos y prioridades de estas partes, y su índice de desempeño, lo que permite a las empresas mejorar su competitividad.
\end{abstract}

Palabras clave: Logística interna, modelo diagnóstico, desempeño, competitividad.

\begin{abstract}
The market has required of the organizations, efficiency, effectiveness, creativity and agility, to be competitive and have their strategies aimed at the sustainability of business defined. In this context, logistics emerges as one of the important links in corporate strategies. However, in prior reading about this topic, there is a lack in the literature about the organization and evaluation of internal logistics. Looking to fill this gap, this paper aims to identify the importance of evaluating internal logistics, identifying its component parts and how they are structured and measured, based on the literature and answers of experts in this field. The degree of importance of these parts will be evaluated by three companies, using a Likert scale. The identification of the component parts of the internal logistics, the weights of the priorities of these parts, and its performance index are showed, which enable the companies to improve their competitiveness.
\end{abstract}

Keywords: Internal logistics, diagnostic model, performance, competitiveness.

1 Departamento de Ciencia Sociales. Universidad Estadual del Amazonas. Brasil. E-mail: orlempinheiro@ gmail.com

2 Departamento de Administración. Universidad Federal del Amazonas. Brasil. E-mail: sbreval@ gmail.com

3 Departamento de Logística y Transporte. Universidad Federal de Santa Catarina.

Brasil.E-mail: taboada@deps.ufsc.br; neimar.follmann@gmail.com

* Autor de correspondencia 


\section{INTRODUCCIÓN}

El advenimiento de la globalización promovió en las organizaciones la busca incesante de la competitividad, obligando a las empresas a salir de un estado de ventajas comparativas a uno de ventajas competitivas. Todavía, las organizaciones que están en la vanguardia de sus sectores y que son consideradas exitosas son aquellas que efectivamente desarrollan sus competencias esenciales procurando ofrecer un patrón de excelencia en bienes y servicios y se preocupan por su estrategia y por la fuerza de trabajo. El mercado ha exigido de las organizaciones un conjunto de características que abarcan: eficiencia, eficacia, dinamismo, creatividad, agilidad, flexibilidad y que posean visión holística, que sean competitivas y que tengan sus estrategias definidas, buscando la sustentabilidad del negocio.

Las organizaciones en lugar de concentrarse en las condiciones del sector, deberían concentrarse en las competencias esenciales de sus empresas y utilizar las habilidades, los procesos y las tecnologías para crear ventaja competitiva sustentable en su cadena de valor. Desarrollando y manteniendo competencias es la manera por la que los dirigentes conseguirán sostener las ventajas competitivas de sus organizaciones [1-3].

Bowersox y Closs [4] destacan los indicadores que evalúan la eficiencia de las actividades y procesos internos, y sugieren indicadores de desempeño logístico clasificados en las siguientes categorías: gestión de activos, costo, productividad, calidad y servicio al cliente. No obstante, es necesario desarrollar una forma de evaluación de desempeño para la cadena de suministros, utilizándose conjuntamente, indicadores externos e internos para evaluar el desempeño de toda la cadena, no solo indicadores internos de logística, de modo que, trabajando en conjunto, las empresas consiguen alcanzar el mejor retorno del negocio de la cadena de suministros a que pertenecen.

En los últimos 20 años se observa la importancia y destaque que la logística viene alcanzando, así como su evolución, principalmente impulsada por el avance de la tecnología de la información, nuevas técnicas de gestión, etcétera.

El proyecto del sistema de logística interna es, por tanto, un aspecto que influye fuertemente en la competitividad del sistema y está, por consiguiente, relacionado con el objetivo de este trabajo.

De acuerdo con [4], el ciclo de actividades de apoyo a la manufactura está directamente relacionado a la logística interna, es decir, a la planificación y control de la producción. De esta forma, el apoyo logístico a la producción, busca principalmente establecer y mantener un flujo económico y ordenado de materiales, así como de stocks en proceso con la finalidad de cumplir las programaciones del sector de producción. La logística de apoyo a la producción tiene como responsabilidades operacionales las siguientes: traslado y almacenaje de los productos, materiales, componentes y piezas semiacabadas.

Según [5], hay una falta de formas estandarizadas de lidiar con las exigencias de la logística interna, lo que influye en el desempeño logístico total. En general, los gestores de la logística están intentando usar las mediciones para ayudarse a proyectar y gestionar sistemas de logísticas más eficientes y eficaces para el cliente. Identificar el valor de la logística interna y sus criterios de desempeño críticos puede ser una manera de ayudar a este desarrollo. Un sistema de logística interna bien concebido y correctamente utilizado aumenta la eficiencia de una organización [6] . Este trabajo de investigación que se presenta aborda aspectos importantes en la concepción de la logística interna, identificando sus partes componentes, cómo medirlas y cómo mejorar estas medidas.

\section{ANÁLISIS TEÓRICO}

\section{Definiciones de logística}

En [7-8], se considera la definición de la logística como "administración de la logística". Cita también que en este concepto puede estar incluido el servicio al cliente, tráfico y medios de transporte, almacenaje, selección del local para fabricar y almacenar, control de inventario, procesamiento de pedidos, compras, traslado de materiales, distribución, abastecimiento de piezas, embalaje, devolución de mercancías y previsión del volumen de pedidos y que una organización debe ofrecer productos y servicios a los clientes de acuerdo con sus necesidades y exigencias del modo más eficiente posible.

En [9-10] relaciona la logística con el marketing, definiéndola como el proceso de gestionar de manera 
estratégica la adquisición, traslado y almacenaje de materiales, piezas y productos acabados, juntamente con el flujo de informaciones, por medio de la empresa y de sus canales de marketing, de modo de maximizar el lucro, actual y futuro, mediante el atendimiento de los pedidos con un bajo costo. No existe una única definición para conceptualizar la logística, que sea aceptada por todos los investigadores de la materia. Lo importante es que las empresas sepan que ella está presente en el mundo empresarial y que los profesionales deben entender su objetivo, que "es tornar disponibles productos y servicios en el lugar donde son necesarios, en el momento en que sean deseados" [9].

La logística puede ser considerada la gestión estratégica de la adquisición, traslado y almacenaje de materiales y productos acabados, sus informaciones relacionadas, mediante el canales de distribución, maximizando el lucro presente y futuro [9].

En resumen, la logística tradicional se refiere a actividades como embalaje, transporte, carga, descarga y almacenaje, etc. Además, la logística moderna reafirma el concepto de gestión de logística integrada y su implementación. Es importante resaltar que la logística moderna debe ser entendida como el medio para la adquisición, la producción y la operación de todo el proceso hasta la entrega al consumidor.

\section{Evolución de los conceptos de la logística}

Las empresas están buscando tornarse cada vez más competitivas en el mercado mundial. Para eso es preciso implementar soluciones eficientes, las que permitan reducir los tiempos y ciclos, el consumo de recursos naturales y además, aumentar la calidad; a fin de superar a los competidores mundiales y conquistar los clientes, cada vez más exigentes en el mercado mundial globalizado [11].

En el ámbito organizacional contemporáneo, la logística aparece como un concepto estratégico, no solo por causa de la gestión de materiales y de la distribución física, sino también por suministrar valores de tiempo y lugar para los clientes, por tornarse un elemento que se distingue para las organizaciones, con agilidad, flexibilidad e integración de sus canales internos y externos. Varios autores describen que el concepto de logística puede ser separado en tres puntos básicos: alimentación (suministradores), plantas (interno) y distribución (clientes), con la agregación de otras varias subactividades, que representan un grupo que es muchas veces definido como altamente empírico, lo que resulta en efectos negativos que influyen directamente en el resultado del desempeño final de las organizaciones, herramientas que son necesariamente decisivas para monitorear su situación [12-13]. La evolución del concepto de logística, se divide en fases, que pueden ser visualizadas en la Figura 1.

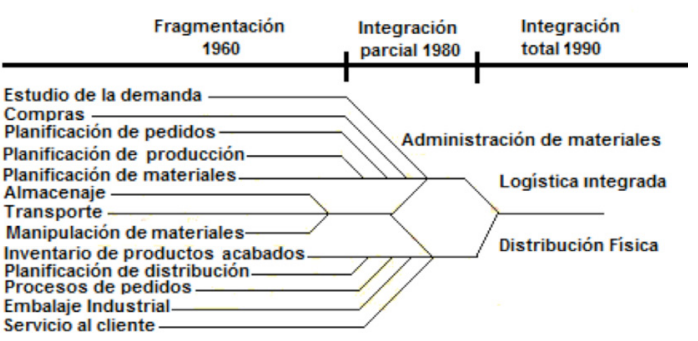

Figura 1. Evolución de los conceptos de la logística. Fuente: [14] adaptado por los autores.

Con el paso del tiempo, ha habido cierto grado de variación del concepto de logística, que va desde un concepto estrecho de distribución física hasta el concepto más amplio de logística. En 1991, el Council of logistics Management [15] formalmente estableció el concepto de logística, y la distribución física ya no se consideró más como logística totalmente sino como una parte de la logística. Por otra parte, la logística moderna es definida como: el proceso de planificación, implementación y control de la eficiencia, el flujo efectivo y almacenaje de mercancías, servicios, e informaciones relacionadas desde el punto de origen hasta el punto de consumo, con el propósito de adecuarse a la exigencia del cliente [16]. Esta definición hace énfasis en la satisfacción del cliente, las actividades logísticas eficientes y la logística extendida desde la fuente de origen hasta la logística de ventas, pasando por la logística de abastecimiento y la logística empresarial (ver Figura 2).

No obstante hay una serie de definiciones comúnmente aceptadas de logística hoy en día. Una definición muy completa y simple es la de las "7 Cs de la Logística", que define la logística como asegurar a disponibilidad del producto correcto, en la cantidad 
correcta y condición correcta, en el lugar correcto, a la hora correcta, para el consumidor correcto, al costo correcto. Una segunda definición es aquella que fue adoptada por el Council of Logistics Management [15], que define la logística como: la parte del proceso de gestión de la cadena de suministros que se ocupa efectivamente de planear, implementar, y monitorear el almacenamiento y flujo directo y reverso de bienes, servicios y todas las informaciones relacionadas a ellos, entre el punto de origen y el punto de consumo, con el objetivo de satisfacer las expectativas del consumidor. Igualmente, en [17] se declara: Logística es el proceso de gestión estratégica de la adquisición, traslado y almacenaje de materiales, piezas y stocks finales (y los flujos de información relacionados) por medio de la organización y sus canales de marketing, de tal forma que la rentabilidad actual y futura sean maximizadas mediante el cumplimiento rentable de órdenes. Finalmente, una definición mucho más simple de logística es la ofrecida en [18], que es la siguiente: Logística es la gestión de inventario en movimiento y en reposo y el objetivo del gerente de logística es lograr alcanzar el menor nivel de inversión en inventario de modo de asegurar la atención al cliente y el mantenimiento de una producción eficiente.

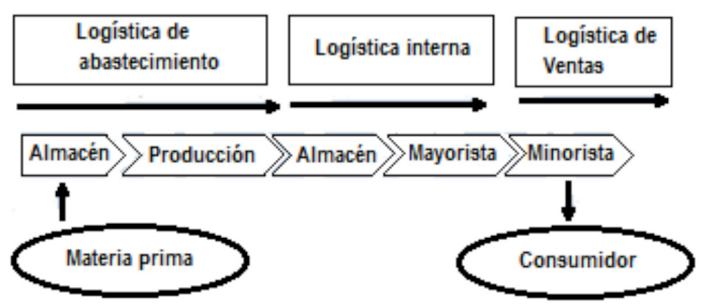

Figura 2. Extensión de los conceptos de la logística moderna. Fuente: Adaptada por los autores de [19].

\section{Logística de entrada}

Las rutas o protocolos de entrega basadas en las redes de logística de entrada (inbound logistics) son usadas por varias empresas cuyos abastecedores están distribuidos ampliamente para reducir los costos mediante la consolidación de mercancías transportadas para un mismo destino.

Gestionar los flujos de materiales en esas redes es una tarea compleja, especialmente si se deben concentrar los efectos de sinergia en el ramo principal para reducir los costos y la polución ambiental. Una técnica para disminuir la sobrecarga en una dirección es el uso de perfiles de entrega, que proveen una frecuencia de entrega fija para cada abastecedor y facilitan la planificación para los socios de la cadena de suministros. La selección de un perfil de entrega tiene efectos tanto sobre el resultado económico como ecológico del sistema de transporte y, por tanto, debe ser hecha cuidadosamente.

\section{Integración de la logística}

La logística integrada es el estado maduro de desarrollo del sector de logística. Desarrollando la industria de logística y mejorando el sistema de logística, el sector de logística se torna un líder en la cadena de producción y coordinación, y es capaz de proporcionar a la comunidad una gama completa de servicios de logística. El desarrollo de la integración logística puede ser dividido en tres niveles: la logística autointegrada, la logística macrointegrada y la logística microintegrada [20].

Antes de la existencia del concepto de logística integrada (abastecimiento-producción-distribución), una parte de las responsabilidades logísticas de hoy estaban subordinadas a la producción o el control de marketing. Es decir, hay una consciencia creciente de que esta función tiene un impacto y participación en las decisiones estratégicas de las organizaciones. "La idea de que las empresas pueden desarrollar capacidades logísticas que soporten los negocios básicos u objetivos de producción no es nueva. A lo largo de la década de 1990, una serie de trabajos han intentado desarrollar tipologías de estrategia de logística que se relacionan con prácticas de empresas individuales para especificar dichas estrategias" [21].

La gestión de éxito de la logística integrada une todas las actividades de logística conjuntas en un sistema que trabaja para minimizar los costos totales y mantener el nivel de servicio deseado por el cliente [22]. Es necesario afirmar que, el costo total incluye cinco categorías principales de costos de logística, que son: niveles de servicios a los clientes, costos de transporte, costos de almacenaje, costos de alta cantidad de stocks y costos de manutención de inventario. Además de eso, el flujo de informaciones desempeña un importante papel en la integración de la logística. 


\section{Sistemas de información de logística}

Los pedidos de los clientes, requisitos de stocks, órdenes de servicio de almacén, documentación de transporte y facturas son los tipos más comunes de informaciones de logística. El uso de la tecnología de sistemas de información logística hizo el intercambio de informaciones de logística más eficiente, eficaz y rápida.

Los sistemas de información de logística son los hilos que unen las actividades logísticas en un proceso integrado. La integración se basa en cuatro niveles de funcionalidad: transacción, control de gestión, análisis de decisión y sistemas de planificación estratégica [23].

Los recursos logísticos son un componente del sistema de logística. Como tal, ellos son un aspecto importante a considerar cuando se evalúa el desempeño. Son los recursos los que permiten el flujo de informaciones y bienes, ya que ellos son los que realizan los procesos y actividades de logística. Cuando se discute acerca de recursos en el área de logística, los recursos físicos (como equipamientos), muchas veces ocupan el primer lugar. No obstante, ya que la manipulación de la información es una parte importante de la logística, los sistemas de Transmisión de la Información (TI) son un recurso importante [24].

\section{Cadena de suministros}

Al pensar sobre la definición de cadena de suministros, existen algunos aspectos que deben tenerse en cuenta. En primer lugar, la corriente de alimentación está formada por varias entidades, que forman un sistema complejo. En segundo lugar, las actividades que son implementadas en esas entidades deben ser tenidas en cuenta. Debido a estas actividades, puede ser activado el flujo de bienes y la atención al cliente en la cadena de abastecimiento. En tercer lugar, la cadena de abastecimiento o suministro debe ser tratada como un sistema entero, las relaciones por medio de las entidades deben ser consideradas, como la relación de interacción, relación de dependencia y de la oferta y demanda del producto acabado y servicio. Por tanto, la cadena de suministros se define como: el sistema de organizaciones, personas, tecnología, actividades, informaciones y recursos involucrados en el traslado de un producto o servicio desde el abastecedor al cliente. Las actividades de la cadena de suministros transforman los recursos naturales, materias primas y componentes en un producto acabado que se entrega al cliente final.

No obstante, el concepto representa una red de organizaciones que trabajan en conjunto para controlar, gestionar y mejorar el flujo de materiales e informaciones de abastecedores para los usuarios finales. Como resultado, el término "red de abastecimiento" sería más apropiado, pero sin embargo no es el más ampliamente aceptado y usado [17]. Independientemente de analizarlo como una cadena o como una red, uno dos factores más importantes de éxito para la cadena de abastecimiento es verla como una única entidad [25], no dispersa y dividida sobre límites funcionales y organizacionales.

\section{Gestión logística}

La gestión de la logística tiene muchos nombres, entre ellos: gestión logística, logística Empresarial, canal de gestión de materiales, distribución física, logística industrial-sistemas de respuesta rápida, gestión logística de la cadena de suministros, etc. [26]. Dentro de todos los nombres citados encima, el término más usado es el de gestión logística "Council of Logistics Management Professionals", es una asociación que es líder mundial, y define la gestión de logística como: la parte de la gestión de la cadena de suministros que planea, implementa y controla el flujo hacia adelante y hacia atrás, y el almacenamiento eficiente de bienes, servicios e informaciones afines entre el punto de origen y el punto de consumo, a fin de atender las exigencias de los clientes [15].

Esta definición incluye el flujo de bienes, servicios e informaciones, tanto en los sectores de manufactura como en los de servicios. Por eso, es fácil de ver que, las características de la gestión de logística son:

- Alcanzar la satisfacción del cliente como primer objetivo.

- Concentrarse en todo el canal de logística.

- Optimizar como propósito las empresas en su totalidad.

- Vincular efectivamente al abastecedor con el usuario final por medio de la tecnología de la información como fuerza motriz.

\section{Eficiencia y eficacia}

El desempeño y la productividad están fuertemente relacionados, y pueden ser divididos en los conceptos de 
eficiencia y eficacia. La eficiencia está relacionada con la utilización de los recursos. Ella lidia principalmente con la entrada del índice de productividad [27]. La eficiencia se refleja, por tanto, en el desempeño de la unidad interna estudiada [6]. La eficacia, por otro lado, está orientada para los resultados y relacionada a ofrecer a los clientes lo que ellos exigen y puede ser asociada al desempeño externo [27]. Simplificada en [28], la eficacia es "hacer las cosas ciertas", mientras que eficiencia significa "hacer ciertas las cosas". Los conceptos de eficiencia y eficacia son bien ilustrados en un modelo [29], como se muestra en la Figura 3.

Para los efectos del presente trabajo, la eficiencia es definida como una medición del desempeño interno (o sea, cuan bien son utilizados los recursos gastados). Mientras tanto, la eficacia se define como una medición del desempeño externo (es decir, lo bien que se alcance la salida deseada).

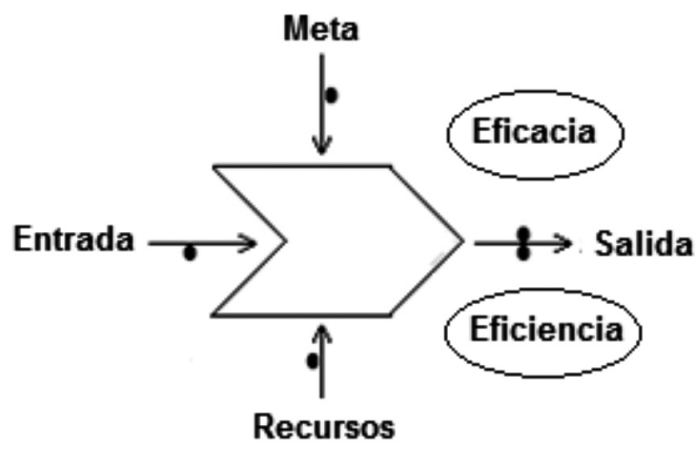

Figura 3. Eficiencia y eficacia. Fuente: Adaptada por los autores de [29].

A pesar de que la eficiencia es una medida de cuán bien son utilizados los recursos gastados [30], no hay ninguna ventaja en tener un elevado grado de eficiencia, si los recursos son usados para alcanzar algo no ligado al objetivo de la organización o sistema estudiado (en otras palabras, alta eficacia). Es la combinación de alta eficiencia y eficacia la que conduce a un alto desempeño. Así, los conceptos de eficiencia y eficacia precisan ser tenidos en cuenta simultáneamente [24]. Esto debe ser considerado, por tanto, un aspecto de preferencia y una parte de las operaciones de logística.

Una forma de analizar o evaluar la eficiencia y la eficacia de las acciones tomadas y tareas ejecutadas es por medio de las medidas de desempeño [31].
Las mediciones de desempeño pueden ser utilizadas tanto para evaluar el desempeño global del sistema como aspectos individuales de comportamientos en diferentes actividades o procesos en el sistema. Así, la medida de desempeño es un instrumento de control. Es también un instrumento que informa a la organización que esfuerzos son recompensados y puede, así, orientar la dirección de una empresa [32].

\section{Desempeño}

Hay muchos motivos para el uso de las medidas de desempeño. Un argumento usado con frecuencia que, de acuerdo con, no puede ser ignorado, es simplemente "lo que es medido, es atendido" [33] [27]. La medición del desempeño es fundamental para el éxito de casi toda organización. Esto es porque crea una comprensión del desempeño de la organización. No obstante, las medidas por si solas no son suficientes. Es importante que la medición de desempeño utilizada realmente mida lo que la empresa precisa saber. También es importante que las medidas de la eficiencia sean acompañadas y evaluadas en relación a los objetivos de la empresa [30]

Lo que debe ser medido, no obstante, difiere de empresa en empresa, dependiendo de sus necesidades y objetivos. La mayoría de las empresas crean su propio sistema de medición de desempeño único, que es un conjunto de medidas de desempeño combinadas para obtener una imagen completa del desempeño de una empresa. A fin de crear un sistema de medición de desempeño exitoso, es vital que los factores clave que influyen en la productividad y desempeño de la empresa sean identificados y considerados [27]. Esto se realiza por dos razones: para evitar el desbordamiento de informaciones irrelevantes y para garantizar que lo que es importante tenga a atención.

En la revisión de la literatura en el área de logística, la importancia de medir para alcanzar un alto desempeño parece indiscutible. Sin embargo, pocas fuentes realmente discuten lo que es el desempeño de la logística. Infelizmente, no hay una respuesta simple, porque el desempeño es un asunto subjetivo. No obstante, la logística trata sobre el abastecimiento y disposición de bienes y todo el mundo concuerda que el recurso clave para la sobrevivencia y para el éxito de la logística es recibir, procesar, recoger, empacar y entregar los productos ciertos, en el precio cierto, en la hora cierta para el lugar cierto [34]. Además, se afirma que debe añadirse que sin daños 
y con la documentación apropiada. Se dice que una orden perfecta es aquella que es recibida, procesada, escogida, embalada, enviada, y entregada en el plazo, sin daños y con toda la documentación apropiada.

Una de las características más obvias es el desempeño de la entrega. Este aspecto tiene generalmente dos dimensiones: velocidad y confiabilidad [21]. La velocidad aquí significa disminuir el tiempo de respuesta y los plazos de entrega, así como dar flexibilidad en las operaciones para ser capaz de adaptar rápidamente la organización y responder a las nuevas exigencias. La confiabilidad en la entrega se relaciona con la capacidad de entregar en el plazo de tiempo establecido.

Mientras tanto, el otro aspecto, pero más específico es la precisión en la entrega que puede verse como la capacidad de evaluar las fechas de entrega adecuadas y la entrega siempre en la fecha prometida (ni antes, ni después).

Otro aspecto (tal vez el más visible) del desempeño logístico es la calidad. Defectos, cantidades incorrectas e ítemes erróneos en las entregas son síntomas de problemas de calidad en procesos logísticos [35]. En cierto sentido, la calidad puede ser fácilmente medida por el número de órdenes incorrectas. No obstante, existen también muchos aspectos no cuantitativos a ella relacionados con la ejecución de procesos y operaciones. Esto también muestra un aspecto adicional del desempeño: la robustez. Internamente, procesos robustos ayudan a reducir los costos mediante la eliminación de errores. Ellos también ayudan a aumentar la confiabilidad, haciendo procesos más adecuados y correctos. Por tanto, la robustez es el corazón del desempeño logístico [36]. Hay también aspectos económicos de desempeño logístico, como competir con el precio de los servicios. Sin embargo, muchas empresas también tienen metas de costo internas para sus operaciones de logística que muchas veces derivan de la orientación que todas las funciones de logística son percibidas como "centros de costo" y, por tanto, la submeta para la distribución física es minimizar los costos [30].

En última instancia, el aspecto más importante del desempeño de la logística interna es:

(1) tener una comprensión clara de los aspectos más importantes a ser evaluados.
(2) asegurarse de que los requisitos y objetivos relacionados son alcanzados de la forma más eficiente posible.

Así, los aspectos de desempeño priorizados se tornan desde el punto de partida cuando se establecen las metas de logística y son, por tanto, la base de una estrategia de logística [37].

\section{Logística interna}

De acuerdo con el punto de vista de la logística como un cuadro o un enfoque que consiste en varias partes y aspectos, ella es muchas veces descrita como un sistema, que es el punto de vista utilizado en este trabajo para analizarla logística interna como parte del sistema. De acuerdo con [37], el sistema de logística es siempre abierto y en un estado de intercambiar con su entorno. No obstante, los límites del sistema y los subsistemas y componentes incluidos varían dependiendo de diferentes perspectivas.

El sistema estudiado en este trabajo es el sistema de logística interna, donde los límites del sistema corresponden a los límites físicos para la empresa en estudio. La logística interna comprende entonces, actividades de logística dentro de los muros de una organización, por ejemplo transportes internos, manejo de materiales, almacenaje y embalaje [37].

Una perspectiva interna del sistema de logística está, de acuerdo con [37], limitada a una cadena de interna de suministros, con el objetivo de crear eficiencia y minimizando la subutilización dentro de la empresa como un todo. Además, los abastecedores y los clientes son componentes externos en el ambiente del sistema de logística interna, una vez que pueden influir en el sistema de logística, pero no pueden controlarlo [37]. El sistema interno se basa en condiciones internas y metas que dictan como los recursos del sistema son utilizados.

De acuerdo con [33], hay tres ángulos diferentes a partir de los cuales, las operaciones de la logística pueden ser vistas: procesos, recursos y organización. Todos esos aspectos pueden ser vistos como partes del sistema de logística, como el flujo de mercancías e informaciones al ser realizadas por medio de una serie de actividades y etapas llamadas procesos. Además, los recursos se refieren a todos los medios, equipamiento y personal necesario para ejecutar 
los procesos. Finalmente, la organización incluye todos los procedimientos de planificación y control necesarios para ejecutar y gestionar el sistema.

Según [34], el consumidor final, en última instancia determina el éxito o fracaso de las cadenas de abastecimiento. Así, una parte importante del desempeño de la logística está ligada al atendimiento al cliente y ser capaz de responder a sus necesidades y requisitos. Cuando se trata de logística interna como sistema, tanto, el cliente como el proveedor del servicio es alguien de la propia organización. Así al ver la logística interna como un sistema, tanto el prestador de servicios y el cliente son la misma empresa. Como tal, las necesidades y exigencias del cliente pueden ser traducidas para los objetivos internos. Por tanto, el desempeño de la logística interna está bajo el control de la empresa, y puede suministrar una indicación más directa de los efectos de la relación entre la estrategia, estructura y logística [21].

Varios autores afirman que un sistema de logística interna bien concebido y correctamente utilizado aumenta la eficiencia de una organización [6]. El proyecto del sistema de logística interna es, por tanto, un aspecto que influye fuertemente en la competitividad del sistema y está relacionado con el objetivo de este trabajo.

Proyectar y mejorar el sistema de logística interna comprende la toma de decisiones en diferentes niveles, como los niveles estratégico, táctico y operacional. Como tal, comprende planificación de largo plazo (estratégico) y aspectos de planificación y control (gestión), de corto y mediano plazo [35]. Un sistema de logística interna que funcione bien exige una participación y un entendimiento del sistema en todos los niveles. Los profesionales de la logística deben estar equipados con la experiencia necesaria en funciones esenciales y críticas para su propia empresa y entender completamente como ellas afectan toda la cadena de valor. Las cadenas de suministro son confrontadas muchas veces con la situación en que ellas tienen que aceptar cierto grado de incertidumbre, sin embargo, deben desarrollar una estrategia que les permita ajustar la oferta a la demanda. En general, se puede decir que una estrategia trata acerca de cómo realizar la planificación, lo que es muy diferente del hacer [35]. La estrategia consiste en esos planos o patrones de acciones que integran los principales objetivos, políticas y actividades de una organización en un todo coherente. Así, una estrategia de logística consiste en esos planos o patrones de acciones que se relacionan con el flujo de materiales.

Una estrategia bien formulada ayuda a utilizar todos los recursos de una organización y crear valor con base a su competencia interna y deficiencias con relación al ambiente externo. No obstante, es de gran importancia que tanto la función logística como la estrategia logística estén integradas y alineadas con otras funciones y estrategias de la organización, a fin de crear competitividad [37].

\section{RESULTADOS Y DISCUSIÓN}

\section{Nueva definición de logística interna}

En el ambiente de fabricación, ha habido una gradual mudanza en el control del mercado del producto por parte de los productores para los clientes y, de ahí para los consumidores finales. Con el aumento de la demanda del cliente, para la mayoría de las industrias de fabricación, se tornó cada vez más importante optimizar el desempeño del proceso industrial. En el contexto de la gestión de sistemas de manufactura, un papel decisivo es jugado por la logística, especialmente la logística interna que puede ser definida como la planificación de procesos y de la organización con vista a optimizar el flujo de materiales e informaciones dentro y fuera de la empresa para maximizar el lucro [38].

Con base a las lecturas de los artículos revisados y la elaboración de un patrón fue posible definir un concepto de la logística interna más abarcador:

\section{La logística interna es la planificación, la ejecución $y$ el control del flujo físico y de informaciones internas de la empresa, buscando la optimización de los recursos, procesos y servicios con la mayor economía posible.}

\section{Procedimiento desarrollado para evaluar la logística interna e identificar sus partes componentes}

En la actualidad existen pocas tentativas de propuestas y técnicas sistemáticas que, mejorando el sistema de manufactura y la logística interna así como el desempeño relacionado, sean capaces de evaluar la dinámica de producción y la mejoría correspondiente. 
Con base en las lecturas de los artículos revisados y una encuesta realizada en 10 empresas del polo industrial de Manaos fue posible identificar las partes componentes de la logística interna. La selección de las 10 empresas tiene como base su representatividad dentro del polo industrial de Manaos, que está constituido por 565 empresas, de ellas 102 son del polo de dos ruedas (para motocicletas). Las 10 empresas pertenecen a este polo de dos ruedas. La encuesta contemplaba el criterio de los gestores y directores, sobre cuáles eran las partes componentes de la logística interna. Fueron realizados algunos ajustes mediante interacciones con profesionales de las empresas investigadas, donde las partes componentes: picking, packing y embalaje fueron aglutinadas, en función de las respuestas, que afirmaban que las referidas actividades eran desarrolladas en el mismo ambiente y las partes componentes: lay-out y localización también hacían parte del almacenaje. Estas partes fueron establecidas a mediante el método inductivo-deductivo. En la Figura 4 se muestran de manera resumida las partes componentes de la logística interna.

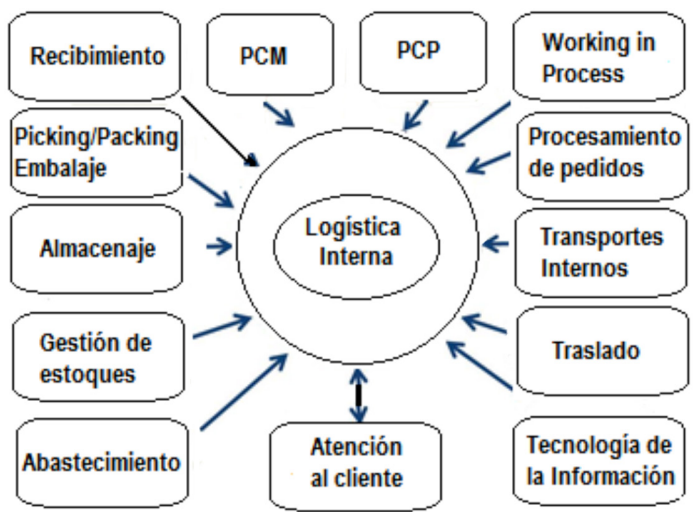

Figura 4. Partes componentes de la logística interna. Fuente: Los autores.

Para evaluar cada parte componente fueron elaboradas 10 preguntas pertinentes y que justificasen la existencia e importancia de cada elemento parte componente de la logística interna, para proponerlas posteriormente a las empresas. En esta etapa también fueron realizados algunos ajustes mediante interacciones con profesionales de las empresas investigadas.

\section{Evaluación del peso de las partes componentes de la logística interna por las empresas}

Para evaluar el peso de cada parte componente de la logística fueron enviadas las mismas a 10 empresas para que las analizaran y le atribuyeran un peso de acuerdo a su importancia en una escala Likert de 1 a 5 , donde 1 tenía poca importancia y 5 mucha importancia de acuerdo con la particularidad y prioridad que las partes componentes representaban para las referidas empresas. En la Tabla 1 se ofrecen los resultados de tres de las empresas estudiadas.

La puntuación máxima del peso que cada empresa puede obtener son 65 puntos, que es el resultado de la multiplicación de los 13 ítemes por la nota máxima de 5 puntos para cada ítemes, cuyo máximo valor es obtenido conforme a la escala Likert. Se observa que la empresa 1 le atribuyó una nota muy baja para los ítemes: almacén, wip y transportes internos, mientras las empresas 2 y 3 le atribuyeron notas 5,5 y 4 , respectivamente para los mismos ítemes.

Se elaboró también en esta tabulación de datos una media aritmética de las tres empresas y se percibió que de la máxima puntuación posible de 65 puntos, la empresa 1 obtuvo 35 puntos, seguido de 61 puntos por la empresa 2 y finalmente la empresa 3 , con 59 puntos, siendo la media aritmética de 51,67 puntos.

El peso es el resultado de la nota o evaluación dada al grado de prioridad (entre 1 y 5) de cada parte componente, dividido por la suma total de los puntos obtenidos por la empresa.

\section{Evaluación de los índices de cada propiedad de} la logística interna por las empresas

En base a la literatura analizada se desarrolló la estructura del modelo de diagnóstico de las partes componentes de la logística interna, su llenado, prueba y validación posterior. Fueron presentadas 10 preguntas para evaluar cada parte componente y se realizó una encuesta en las empresas. Cada parte componente (13) tiene entonces 10 propiedades, donde cada una de estas propiedades se evalúa entre 1 y 5 basado en la escala Likert. El valor máximo de puntuación alcanzar por la suma de los puntos de todas las propiedades es de 50 puntos, lo que constituye el denominador de la fórmula (2).

Estas preguntas fueron también elaboradas con base a la revisión bibliográfica, y de acuerdo con 
Tabla 1. Respuestas de las empresas sobre el grado de importancia de los elementos de la logística interna.

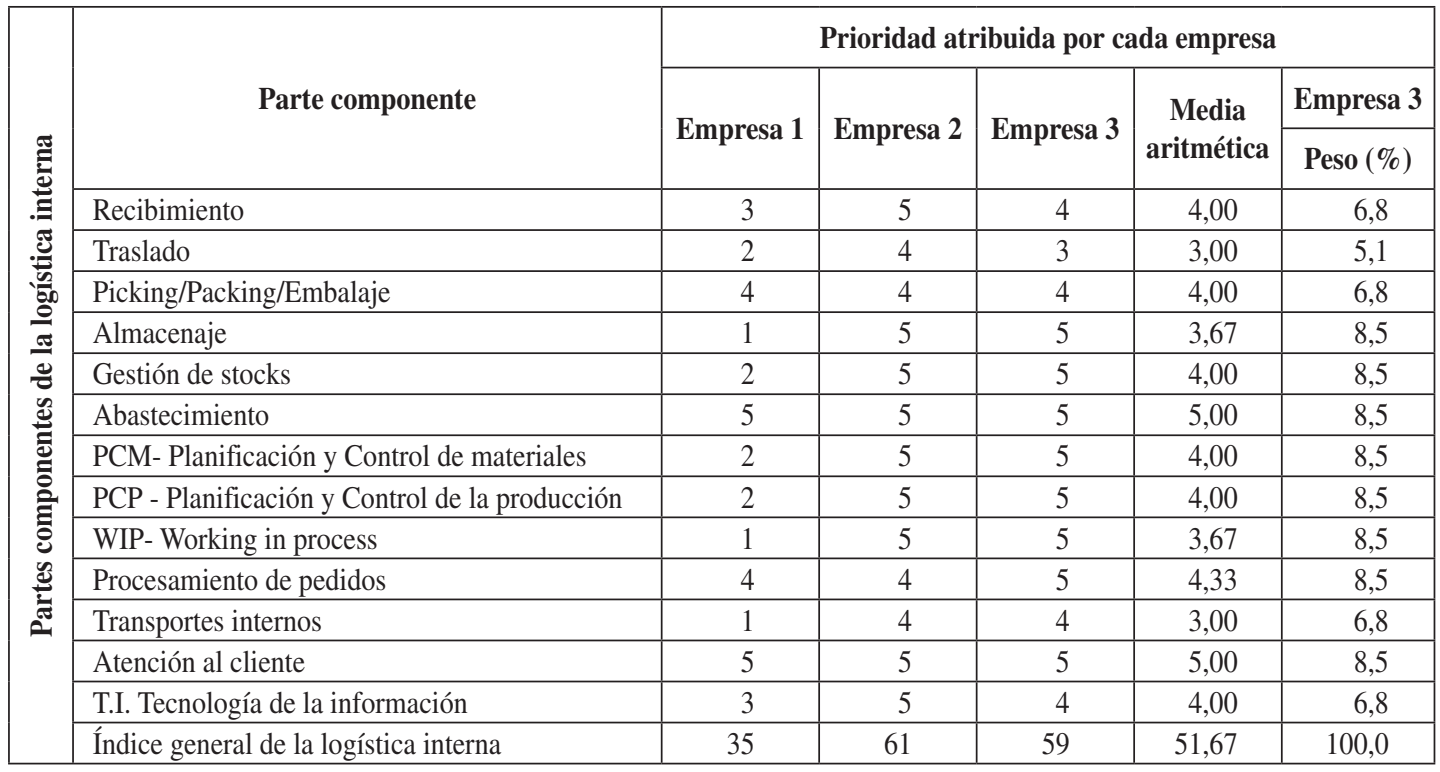

Fuente: Los autores.

los criterios de especialistas de gestión logística de equipos de consultoría y directores de las empresas. Fue desarrollado un tabulador excel para evaluar el desempeño de cada una de las partes componentes de la logística interna, así como el índice de la logística interna de una empresa. El tabulador excel tiene como base las ecuaciones siguientes:

$$
I L I=\sum_{i=1}^{13}\left[\frac{Z_{i}}{100}\right] \cdot W_{i}
$$

Donde:

ILI = Índice general del desempeño de la logística interna;

$W_{i}=$ Peso atribuído a cada parte componente $i$;

$i=$ Cada una de las propiedades analizadas;

$Z_{i}=$ Valor alcanzado en $\%$ por la propiedad $i$ basado en la suma de todos los valores otorgados a cada parámetro de la correspondiente propiedad de la escala Likert de 1 a 5 y dividido entre el valor máximo posible a alcanzar y expresado en $\%$ o sea:

$$
Z_{i}=\sum_{j=1}^{10}\left(\frac{P_{j} \cdot L_{j}}{50}\right) \cdot 100
$$

Donde:

$P_{j}=$ Cada uno de los parámetros que evalúan la propiedad $Z_{i}$ (siempre va a tomar el valor 1 en la expresión anterior);
$L_{j}=$ Valor otorgado al parámetro $P_{j}$ en la escala Likert de 1 a 5.

Fue escogida de forma aleatoria la empresa 3 para responder los cuestionarios elaborados referentes a los 13 elementos o partes componentes de la logística interna. La referida empresa llenó el tabulador excel, obteniendo una puntuación en \% de cada propiedad (entre 0 y 50\%) que fue multiplicada por los pesos ponderados de la Tabla 1 de acuerdo con el grado de prioridad, llegándose a un índice general de la logística interna del 79,17\% como se muestra en la Tabla 2.

\section{CONCLUSIONES}

Esta investigación ofrece varios resultados como contribuciones para la literatura y para el medio empresarial. Como principal resultado ofrece un modelo para la evaluación de la logística interna en las empresas utilizando un indicador denominado índice de logística interna.

Los resultados encontrados para la muestra de 10 empresas, del polo industrial de Manaos, circunscritos en la logística interna de cada una, demuestran la perspectiva de medir el ILI a partir de las partes componentes y como las empresas definen sus prioridades. 
Tabla 2. Índice general de la logística Interna de una empresa.

\begin{tabular}{|c|c|c|c|c|}
\hline \multirow{16}{*}{ 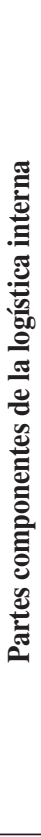 } & \multirow[b]{2}{*}{ Parte Componente } & \multicolumn{3}{|c|}{ Desempeño } \\
\hline & & $(\%)$ & $\begin{array}{c}\text { Peso } \\
(\%)\end{array}$ & Puntos \\
\hline & Recibimiento & 96,00 & 6,8 & 6,53 \\
\hline & Traslado & 88,00 & 5,1 & 4,49 \\
\hline & Picking/Packing/Embalaje & 90,00 & 6,8 & 6,12 \\
\hline & Almacenaje & 86,00 & 8,5 & 7,31 \\
\hline & Gestión de stocks & 86,00 & 8,5 & 7,31 \\
\hline & Abastecimiento & 46,00 & 8,5 & 3,91 \\
\hline & $\begin{array}{l}\text { PCM- Planificación y Control } \\
\text { de materiales }\end{array}$ & 94,00 & 8,5 & 4,62 \\
\hline & $\begin{array}{l}\text { PCP - Planificación y Control } \\
\text { de la producción }\end{array}$ & 92,00 & 8,5 & 4,62 \\
\hline & WIP- Working in process & 88,00 & 8,5 & 7,48 \\
\hline & Procesamiento de pedidos & 88,00 & 8,5 & 7,48 \\
\hline & Transportes internos & 90,00 & 6,8 & 6,12 \\
\hline & Atención al cliente & 88,00 & 8,5 & 7,48 \\
\hline & T.I. Tecnología de la información & 84,00 & 6,8 & 5,71 \\
\hline & $\begin{array}{l}\text { Índice general de la logística } \\
\text { interna }\end{array}$ & & & \\
\hline
\end{tabular}

Fuente: Los autores.

En el trabajo se definieron un conjunto de propiedades para evaluar la logística interna de una empresa. Estas propiedades permiten medir el nivel real de la logística interna de las empresas. Cada parte componente fue evaluada por un grupo de indicadores que permitieron por medio del uso de la escala Likert establecer el índice de logística Interna de una empresa.

Fue establecida una ecuación matemática para determinar este indicador; este procedimiento se considera también como un resultado importante del trabajo. Los resultados de las correlaciones entre los indicadores y la definición de los pesos de los factores para evaluar la logística interna son aspectos inéditos en la literatura consultada. A pesar de estos resultados ser aplicados a determinadas empresas, el modelo analítico que surgió de este proceso puede ser considerado una contribución a la comunidad científica y también al medio empresarial, debido a su posibilidad de adaptación a cada situación.

Finalmente, el procedimiento metodológico para la definición del modelo analítico, conteniendo todos los pasos para la réplica del trabajo se torna un resultado tan importante como la propia aplicación del modelo desarrollado en el trabajo.

Este trabajo a pesar de haber sido aplicado a un pequeño número de empresas, puede ser extendido a un número mayor. Para las futuras investigaciones podría tomarse en cuenta a los abastecedores de suministros, la logística externa, medir el desempeño del sistema logístico: calidad, productividad, etcétera.

\section{AGRADECIMIENTOS}

Al Instituto de Tecnología y Educación Galileo del Amazonas (ITEGAM), a la Universidad Federal de Santa Catarina (UFSC) y a la Universidad Estadual do Amazonas (UEA) por el apoyo brindado al desarrollo de esta investigación.

\section{REFERENCIAS}

[1] J.A. Wagner III and J.R. Hollenbeck. "Organizational behavior: Securing competitive advantage". Routledge. 2014. ISBN: 1135928819.

[2] R.G. McGrath. "Transient advantage", Harvard Business Review. Vol. 91, pp. 62-70. 2013. ISSN: 0017-8012.

[3] B. Meihami and H. Meihami. "Knowledge Management a way to gain a competitive advantage in firms (evidence of manufacturing companies)". International Letters of Social and Humanistic Sciences. Vol. 3, pp. 80-91. 2014. ISSN: 2300-2697. DOI: 10.18052/ www.scipress.com/ILSHS.14.80

[4] D.J. Bowersox and D.J. Closs. "Logística Empresarial: o processo de integração da cadeia de suprimento". Atlas, São Paulo, Brasil. 2008. ISBN: 9788522428779.

[5] C. Finnsgård. "Assembly processes and materials supply systems design". Institutionen för teknikens ekonomi och organisation, Logistik och transport, Chalmers tekniska högskola. Göteborg, Suecia. 2009.

[6] B. Öjmertz. "Materials handling from a valueadding perspective". Chalmers University of Technology. 1998. ISBN: 9171977139.

[7] R.C. Pascual and I. Ribas. "Some trends and applications of operational research/ management science to operations management". International Journal of Production Management and Engineering 
(IJPME). Vol. 3, pp. 1-12. 2015. ISSN: 2340-4876. DOI: http://dx.doi.org/10.4995/ ijpme.2015.3459

[8] O. Jellouli and M. Abdelkadhi. "Test Logistics Maturity of the Industrial Zone in the Region of Gabes". International Journal of Supply Chain Management. Vol. 2, pp. 2013. ISSN: 2050-7399.

[9] D.J. Bowersox, D.J. Closs, M.B. Cooper and J.C. Bowersox. "Gestão logística da cadeia de suprimentos". AMGH Editora. 2013. ISBN: 8580553180.

[10] F.S. Arbache. "Gestão de logística, distribuição e trade marketing". Editora FGV. 2015. ISBN: 8522511543.

[11] M. Eisler and R. Horbal. "Integration of Supplier and Customer's Production Processes". IFIP International Conference on Advances in Production Management Systems. Springer, pp. 3-10. 2009.

[12] R.H. Ballou. "The evolution and future of logistics and supply chain management". European Business Review. Vol. 19, pp. 332348. 2007. ISSN: 0955-534X. DOI: http://dx.doi. org/10.1590/S0103-65132006000300002

[13] D.J. Bowersox, D.J. Closs and M.B. Cooper. "Supply chain logistics management". McGraw-Hill. New York, NY, USA. 2012. ISBN: 978-0078024054.

[14] R.H. Ballou. "Gerenciamento da Cadeia de Suprimentos: Logística Empresarial". Bookman Editora. 2009. ISBN: 8560031464.

[15] B.J. Gibson, J.T. Mentzer and R.L. Cook. "Supply chain management: the pursuit of a consensus definition". Journal of Business Logistics. Vol. 26, pp. 17-25. 2005. ISSN: 2158-1592. DOI: $10.1002 / j .2158-1592.2005 . t b 00203 . x$

[16] Y.-C. Jim Wu. "Contemporary logistics education: an international perspective". International Journal of Physical Distribution \& Logistics Management. Vol. 37, pp. 504528. 2007. ISSN: 0960-0035. DOI: http:// dx.doi.org/10.1108/09600030710776455

[17] M. Christopher. "Logistics and Supply Chain Management" 4th Edition. Prentice Hall. New York, USA. 2011. ISBN: 978-1-08379-7.

[18] R.V. Delaney. "Trends in logistics and US world competitiveness". Transportation Quarterly. Vol. 45, pp. 1991. ISSN: 0278-9434.

[19] C.J. James, D.F. Wood, D.L. Wardlow and P.R. Murphy Jr. "Contemporary logistics".
New York, USA. Macmillan. 1999. ISBN: 9780137985487

[20] J. Wisner, K.-C. Tan and G. Leong. "Principles of supply chain management: a balanced approach". Cengage Learning. 2015. ISBN: 1285428315.

[21] G.N. Stock, N.P. Greis and J.D. Kasarda. "Logistics, strategy and structure: a conceptual framework". International Journal of Operations \& Production Management. Vol. 18, pp. 37-52. 1998. ISSN: 0144-3577. DOI: $10.1108 / 09600039910273948$

[22] Y. Wang, T. Lu and C. Zhang. "Integrated logistics network design in hybrid manufacturing/remanufacturing system under low-carbon restriction". In: LISS 2012, pp. 111-121. Springer, 2012.

[23] S.E. Fawcett, L.M. Ellram and J.A. Ogden. "Supply chain management: from vision to implementation". Pearson. London, Inglaterra. 2014. ISBN: 1292022191.

[24] E. Sandberg. "Understanding logisticsbased competition in retail-a business model approach". International Journal of Retail \& Distribution Management. Vol. 41, pp. 176188. 2013. ISSN: 0959-0552. DOI: http:// dx.doi.org/10.1108/09590551311306237

[25] V. Florea. "Principles of Modern Logistic Strategies". Ovidius University Annals, Series Economic Sciences. Vol. 12, pp. 2012. ISSN: 1582-9383.

[26] J.R. Stock and D.M. Lambert. "Strategic logistics management". McGraw-Hill/Irwin Boston, MA, USA. 2001. ISBN: 978-0256136876.

[27] C. Kao and S.-N. Hwang. "Multi-period efficiency and Malmquist productivity index in two-stage production systems". European Journal of Operational Research. Vol. 232, pp. 512-521. 2014. ISSN: 0377-2217. doi:10.1016/j.ejor.2013.07.030

[28] D. Sink and T. Tuttle. "Planning and measurement of in your organisation of the future". Norcross, USA. 1989. ISBN: 9780898060904.

[29] D. Katic, V. Majstorovic and I. Colak, "Performance measurement review". Annals of DAAAM \& Proceedings. Vol. 22, pp. 515516. 2011. ISSN: 1726-9679.

[30] J.T. Mentzer and B.P. Konrad. "An efficiency/effectiveness approach to logistics performance analysis". Journal of Business Logistics. Vol 12, pp. 33-62. 1991. 
[31] A. Neely, M. Gregory and K. Platts. "Performance measurement system design: A literature review and research agenda". International Journal of Operations \& Production Management. Vol. 25, pp. 12281263. 2005. ISSN: 0144-3577. DOI: http:// dx.doi.org/10.1108/01443579510083622

[32] M.Zairi. "Measuring performance for business results". Springer Science \& Business Media. 2012. ISBN: 9401113025.

[33] B. Rouwenhorst, B. Reuter, V. Stockrahm. G. Van Houtum, R. Mantel and W. Zijm. "Warehouse design and control: Framework and literature review". European Journal of Operational Research. Vol. 122, pp. 515-533. 2000. ISSN: 0377-2217. DOI: 10.1016/ S0377-2217(99)00020-X

[34] Siddhartha and A. Sachan. "Review of agile supply chain implementation frameworks". International Journal of Business Performance and Supply Chain
Modelling. Vol. 8, pp. 27-45. 2016. ISSN: 1758-9401. DOI: http://dx.doi.org/10.1504/ IJBPSCM. 2016.075999

[35] H. Jiang. "Research on Logistics Management and Strategy in Supply Chain for Efficiency Improvement". Advances in Information Sciences and Service Sciences. Vol. 5, pp. 909-915. 2013. ISSN: 1976-3700.

[36] A. Harrison and R.I. van Hoek. "Logistics management and strategy". Pearson Education. 2005. ISBN: 0273685422.

[37] P. Jonsson. "Logistics and supply chain management". McGraw-Hill. New York, USA. 2008. ISBN: 13-978-0-07-711738-2.

[38] N. Costantino, M. Dotoli, M. Falagario, M.P. Fanti and A.M. Mangini. "A model for supply management of agile manufacturing supply chains". International Journal of Production Economics. Vol. 135, pp. 451457. 2012. ISSN: 0925-5273. DOI: 10.1016/j. ijpe.2011.08.021 